\title{
SPIKE ACTIVITY IN THE CEREBRAL CORTEX
}

\author{
By DR. G. K. SMITH \\ National Physical Laboratory, Teddington, Middlesex \\ AND \\ DR. D. R. SMITH \\ Case Institute of Technology, Cleveland, Ohio
}

$\mathrm{W}$ E have recently been investigating the origin and nature of the spontaneous activity of neurones in the cat's cerebral cortex. The work is an extension in the cerveau isolee of that of Martin and Branch ${ }^{1}$, Girstein and Kiang ${ }^{2}$ in the anæsthetized cortex, who observed that the shorter intervals between successive action potentials fit a random distribution while the longer intervals occur more frequently than such a process would predict. Our results lead us to conclude that at least two independont mechanisms combine to generate the signal which consists of bursts of random activity (that seen by the workers just mentioned) separated by periods of inactivity of variable duration. The preparation used was the unanæsthetized cat isolated forebrain ${ }^{3}$, prepared under ether. Following complete mid-collicular brain-stem transection the animal was allowed to recover from the anæsthetic and recordings of unit activity were made extracellularly using glass micro-pipette electrodes. The unit activity of the unstimulated cortex was stored on magnetic tape and afterwards $2-5 \mathrm{~min}$ samples were analysed using wellknown statistical techniques.

The most useful statistic was found to be the distribution of intervals between successive action potentials plotted semi-logarithmically as in Fig. 1. In this figure each ordinate represents the number of intervals per second equal to or greater in duration than the value of time at the abscissa. A random series of pulses gives a straight line when plotted in this way. We examined records from forty neurones located in all parts of the cortex and at all depths. Fig. $1 A$ is typical of the diagrams obtained in 25 cases (Group $I$ ) and Fig. $1 B$ is typical of the remainder (Group II).

We will deal with Group I first, as it is the simpler case. In this group the intervals of length greater than $300 \mathrm{msec}$ are distributed exponentially, that is, the distribution does not differ significantly from that of a random pulse signal. By the method of maximum likelihood it is possible to determine the most likely exponential constant for the distribution. If the resulting theoretical distribution is subtracted from the observed distribution in the interval range $30-300 \mathrm{msec}$ the residual interval distribution is also best fitted by an exponential. In most cases the intervals between zero and $30 \mathrm{msec}$ are greater in number than would bo expected from an exponential distribution. If wo ignore for the moment this relatively short set of intervals the resulting pair of exponential distributions suggests that the neurone spends part of its time firing at random with a high average frequency (the range from cell to cell is $5-60 / \mathrm{sec}$ ) and another part of its time firing at random with a lower average frequency (range $0 \cdot 2-6 / \mathrm{sec}$ ). Analysis of dependence of successive intervals and of the distribution of the number of action potentials falling within a fixed time confirms that the use of the term 'random' is justified.
By making use of the gradients and zero intercepts of the theoretical distributions obtained by the maximum likelihood method we can determine the time spent by the neurone in the two random processes. In all Group I neurones so far tested the sum of these two times equals the total length of the sample within the experimental error. Hence the switching time from one frequency to the other is very short.

We can now picture the signal as consisting of 'bursts' of high frequency random activity. We may now ask what happens in the intervals between bursts. Consider two models to represent the spontaneous activity: (1) Bursts of relatively high-frequency random activity switched on at random times for unknown and perhaps random periods. The neurone is silent between bursts. (2) Bursts as in (1) superimposed on, or alternating with, a random slow 'background' activity. From these models the intervals of the low-frequency random distribution can be interpreted in two ways: (1) as the intervals between the last pulse of one burst and the first of the next burst, or (2) as intervals between pulses of a slow randomly firing background compounded with intervals of (1) and intervals between pulses at the extremes of a burst and pulses of the 'slow activity'. The analysis of neurone signals from the unstimulated brain cannot distinguish between these two possibilities because they are statistically equivalent.

Stimulation of the brain can change the firing pattern of cortical neurones ${ }^{4,5}$, and it is profitable at this stage to examine these changes in terms of the models. Figs. 2 and 3 show the effects of local currents and electrical stimulation of the cortical surface on neurones of Group I. In each case the stimulation alters only the distribution of

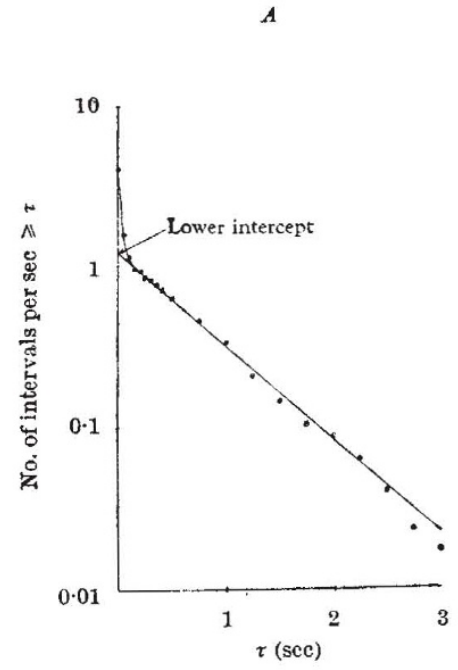

$B$

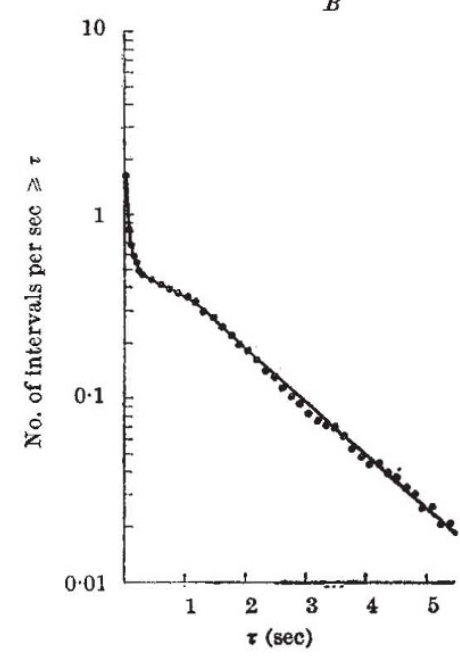

Fig. 1. Interspike interval distribution diagram. $A$, analysis of spontaneous discharge recorded from a single cell in the median suprasylvian gyrus. The ordinate is the number of intervals of duration $\tau$ seconds or longer divided by the total length of the sample (300 $\mathrm{sec})$. The intervals greater than $300 \mathrm{msec}$ are best fltted by an exponential of gradient $1 \cdot 32$. The $\chi^{2}$ test shows that the exponential fits the points at the 25 per cent level of significance, hence the cell is of group I. Subtraction of this exponential from the data leaves a second exponential in the range $30-300$ msec. $B$, cell of group II. Intervals greater than 300 deviation from exponential might be explained by an additional assumption rather than by assuming that the distribution follows a different law 


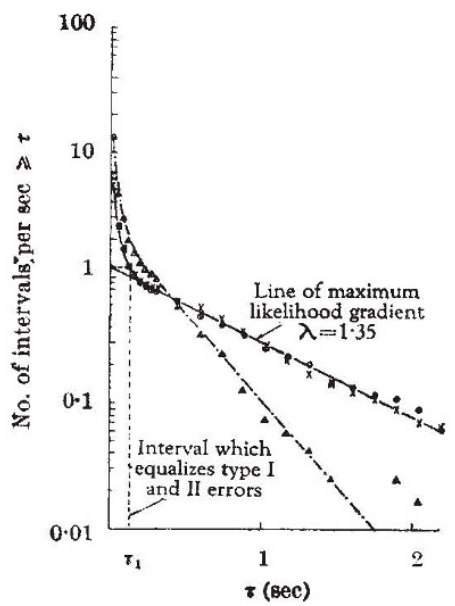

$B$

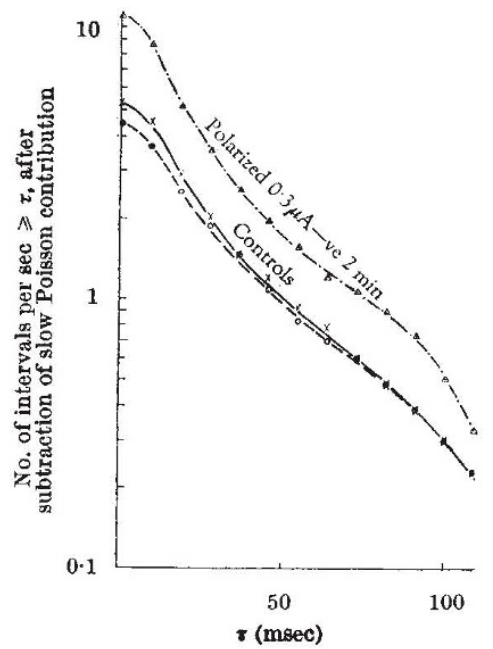

Fig. 2. Effect of local currents on the distribution of interspike intervals. $\triangle$ Spontaneous spike distribution during $2 \mathrm{~min}$ period in which the microelectrode was made negative and passed $0.3 \mu \mathrm{amp}$. $\times$, control before $; \odot$, control after polarization. $\boldsymbol{A}$, complete distribution. Note that the value of the ordinate at $\tau=0$ is the average firing rate. Before polarization it was 5.5 per sec. It rose to 13.4 per sec during polarization and fell to 6.3 per sec when the current was switched off. $B$, short interval distribution after subscribed in the text. Note that during polarization the number of short intervals as deincreases but the exponential gradient is unchanged. 'The observation is interpreted as an increase in the number of burst per unit time with no change in the rate of flring within bursts

$A$

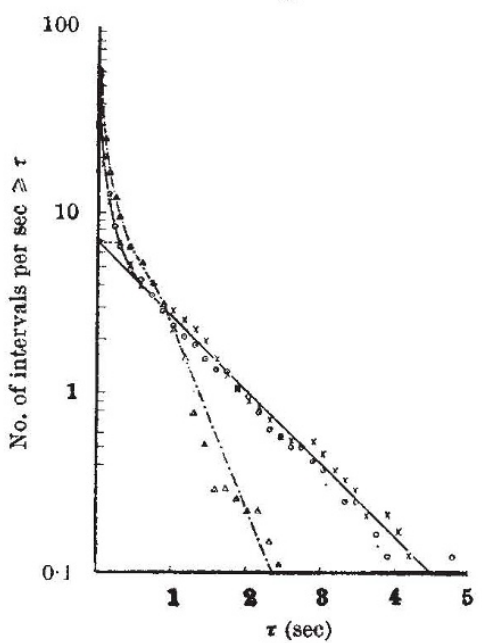

$B$

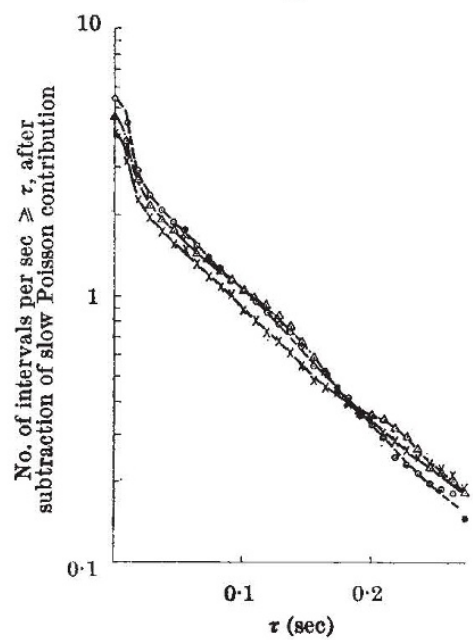

Fig. 3. Effect of rhythmic electrical stimulation of the cortical surface on the spontaneous discharge of a cell in the median suprasylvian gyrus. $\Delta, 5$ min record during stimulation of a point $10 \mathrm{~mm}$ from the recording point on the same gyrus. The stimulus consisted of a cluster of 10 shocks at 100 per sec presented once per second, strength about 75 per cent of maximal for the surface negative response. The cell fired with increased probability following the stimulus 5 . $\times$, control distribution before, $\odot$, after stimulation. $A$, fullinterval distribution. $B$, distribution of short intervals after subtraction of long interval distri-

long intervals leaving the firing rate and distribution within bursts unchanged. The local currents also increase the time spent in bursts as reflected in the elevation of the ourve without change in the slope. Note also that the distribution of long intervals remains exponential although its gradient is increased, that is, the ratio of long to short intervals has been decreased. Tip positive polarization has the reverse effect.

In order to interpret this result in terms of the two models we next looked in more detail at the effects of polarizing current on the distribution of long intervals. In some neurones the gradients of the two random components are sufficiently different to permit recognition of individual bursts. In such cells one can set up a criterion which will, in fact, provide a fairly rigorous definition of bursts. This can be done by choosing an interval such to depth within the cortex. The diagram obtained from a three-minute sample of a given neurone is essentially unchanged when repeated after 20 min or more.

It must be emphasized that the results only apply to intervals greater than 30 msec. Shorter intervals frequently deviate from this picture and may be of physiological importance.

These results are not sufficient to permit conolusions regarding the mechanism of genesis of the signal. Burns ${ }^{4}$

Table 1

\begin{tabular}{|c|c|c|c|}
\hline \multirow{2}{*}{$\begin{array}{l}\text { Minimum No. of } \\
\text { spikes per burst }\end{array}$} & \multicolumn{2}{|c|}{$\begin{array}{l}\text { Gradient of interburst interval } \\
\text { distribution }\end{array}$} & \multirow{2}{*}{$\stackrel{\text { Ratio }}{\text { polarized/control }}$} \\
\hline & Control & Polarized & \\
\hline $\begin{array}{l}4 \\
3 \\
2 \\
1\end{array}$ & $\begin{array}{l}0.46 \\
0.59 \\
0.79 \\
1.28\end{array}$ & $\begin{array}{l}1 \cdot 13 \\
1.55 \\
2 \cdot 27 \\
3.11\end{array}$ & $\begin{array}{l}2 \cdot 46 \\
2 \cdot 63 \\
2 \cdot 87 \\
2 \cdot 43\end{array}$ \\
\hline
\end{tabular}


has shown that bursts in isolated cortex are maintained by a reverberating network of similar neurones. The results reported here make it difficult to see how such a net could be responsible for bursts in the intact cortex, for if the cell possesses classical properties, one would expeot that local polarization would alter the threshold of the cell to synaptic bombardment from the net, thus changing the frequency of firing within bursts. We have compared the lengths of inter-burst intervals with the duration and number of action potentials in the bursts preceding them and found no correlation. Thus the burst mechanism appears to be independent of the mechanism which generates spike discharges during bursts.
A more detailed account of this work proposing a mathematical model of the signal is in the press. Work is in progress which may lead to a further clarification of the mechanisms involved and of the role of very short intervals.

This work has been carried out as part of the research programme of the National Physical Laboratory, and is published by permission of the Director.

${ }^{1}$ Martin, A. R., and Branch, C. L., J. Neurophysiol., 21, 368 (1968),

"Girstein, G. L., and Klang, N. Y.-S., Biophys. J., 1, 15 (1960).

${ }^{3}$ Burns, B. D., and Grafstein, B., J. Physiol., 118, 412 (1952).

- Burns, B. D., J. Physiol., 127, 168 (1955).

'Burns, B. D., and Smith, G. K., J. Physiol., 164, 238 (1962).

\title{
COUPLING OF CYCLIC CHEMOTHERAPEUTIC COMPOUNDS TO IMMUNE GAMMA-GLOBULINS
}

\author{
By Dr. S. DeCARVALHO, Dr. H. J. RAND and A. LEWIS \\ Laboratory of Cancer Virology and Immunology, Rand Development Corporation, \\ Cleveland, Ohio
}

$\mathrm{B}^{\mathrm{x}}$ virtue of their serologicel specificity, immune $\gamma$-globulins may serve as 'guided missiles' to carry and deliver therapeutic agents to target cells containing the speoific antigens. This article deals with some of the chemical problems involved in the coupling of therapeutio agents to $\gamma$-globulins.

The following procedure represents an adaptation of the method utilized by Heidelberger ${ }^{1}$ to couple $\beta$-naphthol 3:6 sodium disulphonate $(R$-salt $)$ to crystalline egg albumin, and by Kabat ${ }^{2,8}$ to couple the same dye to horse serum albumin. Mathé et al. make quick reference to having coupled methotrexate to rabbit hyperimmune $\gamma$-globulin by diazotization", but no details are given.

The method here described was examined in detail and criteria for the end-points of each step of the coupling were developed.

The following materials were used:

Therapeutic compounds. Methotrexate (courtesy of Dr. J. M. Ruegsegger, Lederle Laboratories, Pearl River, New York) ( $A$-methopterin) 4-amino-N ${ }^{10}$-methyl pteroylglut. amic acid (MTX), lot No. 1260-01; uracil mustard (courtesy of Dr. H. Petering, Upjohn Co., Kalamazoo), lot, No. $2358 K$ (UM); 5 -fluorouracil (courtesy of Dr. G. Zbinden, Hoffmann-La Roche, Inc., Nutley, New Jersey), lot No. 009122 (5-FU); tetracycline ('Achromycin V', Lederle), lot No. 4859-23; 6-mercaptopurine hydrate (Nutritional Biochemical Co., Cleveland, Ohio), lot No. 4554 (6MP); chlorambucil (Nutritional Biochemical Co.), lot No. 9981; 'Thio-Tepa' (Lederle), lot No. 4650-91.

Gamma-globulins. Human $\gamma$-globulin from pooled serum (Cutter Laboratories, Berkeley, Calif.), lot No. G6083 (HSGG); horse anti-tumour hyperimmune $\gamma$-globulin, lot No. 257-29 (ref. 5) (HTUGG); horse anti-leukæmia hyperimmune $\gamma$-globulin, lot No. 567-M1-21 (ref. 5) (HLKGG).

Even though there was successful coupling with all the therapeutic compounds listed, methotrexate, uracil mus tard and 5-FU were the only ones investigated in detail.

The coupling develops through the following three steps: (1) diazotization of benzidine; (2) coupling of the therspeutic compound to diazotized benzidine; (3) coupling of the compound obtained in (2) to $\gamma$-globulin.

The temperature of all operations is $7^{\circ}-8^{\circ} \mathrm{C}$. All reagents are added drop by drop with constant stirring.

Step $I$. In a $100-\mathrm{ml}$. beaker place $37.0 \mathrm{ml}$. of benzidine reagent $(720.0 \mathrm{mg}$ of analytical grade benzidine in $6.50 \mathrm{ml}$. of $6 \mathrm{~N}$ hydrochloric acid added to $31.50 \mathrm{ml}$. of distilled water). Cool to $8^{\circ} \mathrm{C}$ in a bath of ice water oversaturated with sodium chloride. Below $6^{\circ} \mathrm{C}$ this reagent freezes. Over a period of 2 min add $14.00 \mathrm{ml}$. of pre-cooled sodium nitrite reagent $(1.30 \mathrm{~g}$ of sodium nitrite in $32.0 \mathrm{ml}$. of distilled water). The end-point was determined in the following way. Immediately after each eddition of 0.1 -

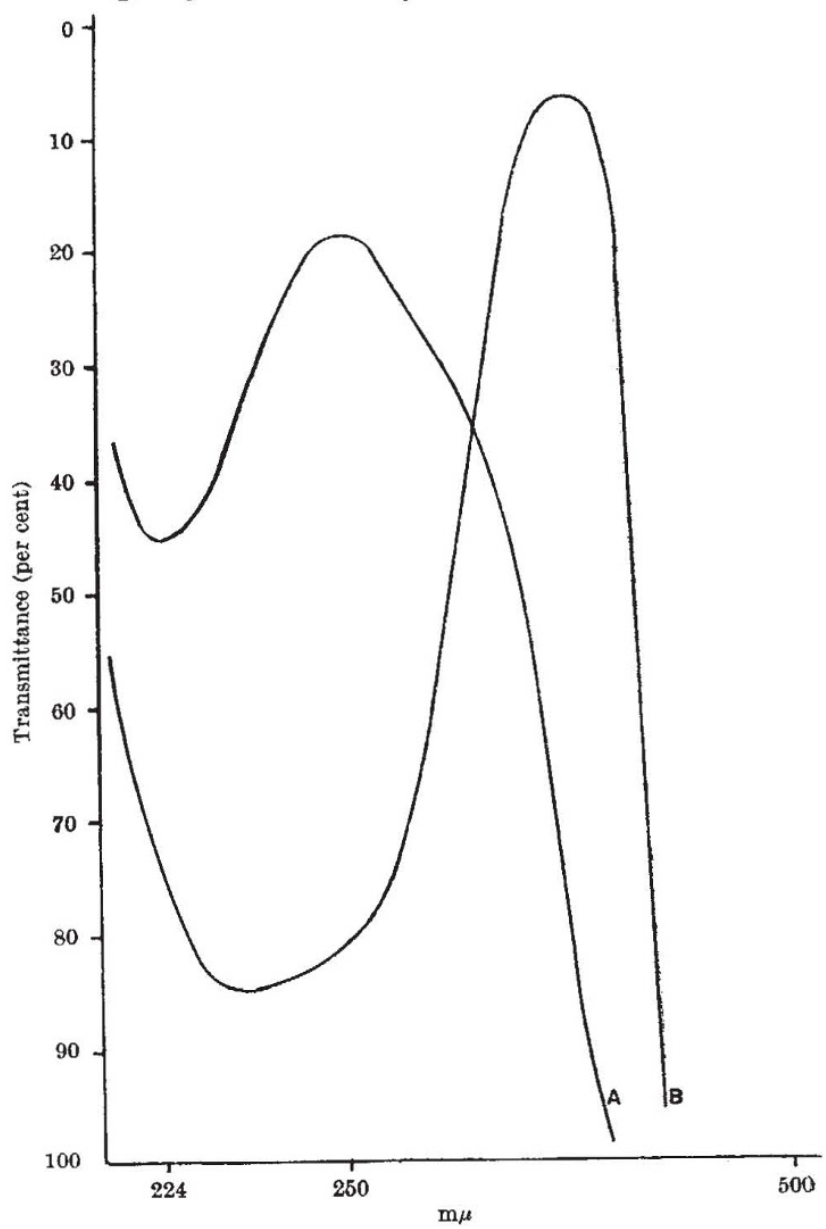

Fig. 1. $A, 2 \mu \mathrm{g} / \mathrm{ml}$. benzidine solution in $6 \mathrm{~N}$ hydrochloric acid at $p$ H $1 \cdot 0$; maximum absorption peak, $250 \mathrm{~m} \mu$; minimum absorption absorption peak, $313 \mathrm{~m} \mu$; minimum absorption peak, $234 \mathrm{~m} \mu$ 